\title{
Diffusion-affected passive scalar transport in an ellipsoidal vortex in a shear flow
}

\author{
K. V. Koshel ${ }^{1,2,3}$, E. A. Ryzhov $^{1}$, and V. V. Zhmur ${ }^{4,5}$ \\ ${ }^{1}$ V.I.Il'ichev Pacific Oceanological Institute, 43, Baltiyskaya Street, Vladivostok, 690041, Russia \\ ${ }^{2}$ Far Eastern Federal University, 8, Sukhanova Street, Vladivostok, 690950, Russia \\ ${ }^{3}$ Institute of Applied Mathematics, 7, Radio Street, Vladivostok, 690022, Russia \\ ${ }^{4}$ P. P. Shirshov Institute of Oceanology, 36, Nahimovski prospect, Moscow, 117997, Russia \\ ${ }^{5}$ Moscow Institute of Physics and Technology, 9, Institutskiy Pereulok, Dolgoprudnyi, Moscow region, 141700, Russia \\ Correspondence to: K. V. Koshel (kvkoshel@ poi.dvo.ru)
}

Received: 30 January 2013 - Revised: 13 May 2013 - Accepted: 25 May 2013 - Published: 1 July 2013

\begin{abstract}
By employing an analytical model for a constantvorticity distributed vortex, namely, the ellipsoidal vortex embedded in a constant buoyancy frequency shear flow, the problem of the passive scalar transport through the vortex's boundary is addressed. Since the model's governing equations do not allow such transition to occur, we implement a low-scale diffusion process into the vortex model. Taking into consideration the diffusion term, we study the passive scalar transport in a steady state (the boundary of the ellipsoidal vortex does not change in time) and in a perturbed state (the boundary of the ellipsoidal vortex changes in time periodically) within the time scope corresponding to the characteristic life cycle of a mesoscale oceanic eddy. An increase of the passive scalar transport through the boundary in the perturbed state in comparison with the steady state due to the irregular dynamics of the surrounding flow is shown. The applicability scopes of the investigation for studying oceanic eddies in nature are discussed.
\end{abstract}

\section{Introduction}

An ellipsoidal vortex is a constant-vorticity distributed vortex model for studying the dynamics of oceanic meso- and submesoscale eddies as a whole and for studying the dynamics of passive scalars comprised within the eddies and their surroundings (Zhmur, 1988, 1989; Meacham et al., 1994). The ellipsoidal vortex model is constructed under the quasigeostrophic approximation and the buoyancy frequency constancy assumption of the surrounding linear shear flow. Al- though the model is quasi-three-dimensional (the ellipsoid vortex has a nonzero vertical scale), the dynamics within the vortex is non-divergent at each horizontal plane, so, the dynamics of each horizontal section of the vortex is similar to the one of the classical Kida vortex (Kida, 1981).

Such an ellipsoidal vortex has the prominent feature of preserving its boundary form in a linear shear flow, being generally motionless or performing periodic or aperiodic motions. However, the surrounding fluid being affected by the boundary change can exhibit irregular dynamics (Polvani and Wisdom, 1990; Dahleh, 1992; Zhmur et al., 2011), namely, the exponential divergence of close trajectories for a finite time, the phenomenon is widely known in fluid mechanics as chaotic advection (Aref, 1984, 2002).

The most evident advantage of this model is an analytically derived stream function that provides an opportunity to obtain some insight into the leading order dynamics, such as the response to external shear, and strain, and the possibility of different motion types (rotation, oscillation, elongation) of vorticity patches in shear flows. However, the possibility to derive the stream function restricts the number of processes that, in one way or another, affect the vortex dynamics in the ocean. The main restriction of this ellipsoidal vortex model is that its governing equations do not allow the fluid particles from the vortex and surroundings to cross the vortex boundary, so, there is no exchange of fluid particles between the vortex and exterior flow. The vortex's boundary in this case plays the role of a barrier that denies scalar transport including the chaotic one through itself (Brown and Samelson, 1994). In order to somehow generate 
such fluid particle exchange, in this paper, we suggest implementing a low-scale diffusion process to the ellipsoid vortex model, so that such fluid particle transition between the vortex and its surroundings can occur.

Thus, the main aim of the paper is to study how the implementation of the diffusion process affects the scalar transport within the vortex and its surroundings. Such a problem is of interest partly since, according to satellite measurements (Chelton et al., 2007, 2011), an oceanic mesoscale eddy although it can last quite a long time it always disappears due to a certain loss of its vorticity (Balasuriya and Jones, 2001; Balasuriya, 2004). This suggests that the eddy's boundary permits the eddy's vorticity to pass through it while the backward flux of the exterior vorticity does not compensate the loss.

\section{Ellipsoidal vortex model}

A detailed derivation of the ellipsoidal vortex model can be found, for instance, in Meacham et al. (1994). Here, we present only the derivation's points that are necessary for the present paper. First, the basic approximations of the ellipsoidal vortex model are the quasi-geostrophic $f$-plane flow in an infinitely deep ocean with a constant buoyancy frequency, $N=$ const. Also, it is worth noting that all the following expressions are already dimensionless (for details see Zhmur et al., 2011). By introducing the length scale, $L^{*}$, the depth scale, $H^{*}$, the velocity scale, $U^{*}$, and the buoyancy frequency scale, $N^{*}$, one can construct the timescale, $T^{*}=\frac{L^{*}}{U^{*}}$, and the stream-function scale, $\Psi^{*}=U^{*} L^{*}$. Given these scales and approximations, the potential vorticity conservation law becomes true in the simplified form (Pedlosky, 1987):

$\frac{\mathrm{d}_{\mathrm{h}}}{\mathrm{d} t} q=0$,

where $q=\Delta_{\mathrm{h}} \psi+\frac{\partial}{\partial z} \frac{f^{2}}{N^{2}} \frac{\partial \psi}{\partial z}$ is the potential vorticity, and $\Delta_{\mathrm{h}}=\frac{\partial^{2}}{\partial x^{2}}+\frac{\partial^{2}}{\partial y^{2}}$ is the horizontal Laplace operator, $\frac{\mathrm{d}_{\mathrm{h}}}{\mathrm{d} t}=\frac{\partial}{\partial t}+$ $u \frac{\partial}{\partial x}+v \frac{\partial}{\partial y}$ with the horizontal velocities, $u$ and $v$, satisfying the geostrophic relations,

$u=-\frac{\partial \psi}{\partial y}, \quad v=\frac{\partial \psi}{\partial x}$.

$\psi$ is the geostrophic stream function that defines unambiguously the dynamics of the flow (Pedlosky, 1987).

In this simplified formulation, the potential vorticity, $q$, is a Lagrangian invariant meaning it can be treated as a passive scalar. Thus, one can choose a certain volume of the fluid whose vorticity is different from that of the surrounding flow, and then study the evolution of this volume. A few volume forms are known to preserve their boundary in a linear shear flow. One of these is the ellipsoidal form which is believed to be of use for comparing its dynamics with the one of the meso- and submesoscale eddies in the ocean (Meacham et al., 1994). Given $a, b$, and $c$ being the semiaxes, $\theta$ is the angle between semiaxis $a$ and the x-axis. The ellipsoid evolves in a linear shear flow, with $e$, and $\gamma$ being the strain and surrounding vorticity components of the linear shear flow. The ellipsoidal form is governed by the following equations (Zhmur, 1988):

$$
\begin{aligned}
& \frac{\mathrm{d} a}{\mathrm{~d} t}=a e \cos (2 \theta), \quad \frac{\mathrm{d} b}{\mathrm{~d} t}=-b e \cos (2 \theta), \\
& \frac{\mathrm{d} \theta}{\mathrm{d} t}=\Omega+\gamma-\frac{a^{2}+b^{2}}{a^{2}-b^{2}} e \sin (2 \theta),
\end{aligned}
$$

where

$\Omega=\sigma a b \tilde{c} \int_{0}^{\infty} \frac{\mu \mathrm{d} \mu}{\left(a^{2}+\mu\right)\left(b^{2}+\mu\right) \sqrt{\xi(\mu)}}$

is the proper rotation of the ellipsoidal vortex without any external flow, $\tilde{c}=\frac{N}{f} c, \xi(\mu)=\left(a^{2}+\mu\right)\left(b^{2}+\mu\right)\left(\tilde{c}^{2}+\mu\right)$, $N=$ const is the buoyancy frequency, $f=$ const is the Coriolis parameter, $\sigma=\alpha-\gamma, \alpha=$ const $\neq 0$ is the ellipsoid vortex's vorticity. System (3) governs the dynamics of the ellipsoidal vortex's boundary. According to Zhmur and Pankratov (1989), the vortex can perform three characteristic types of motion: two periodic - rotation and oscillation, and one aperiodic - infinite elongation. Also, there are parameters when the vortex is aligned along the strain and becomes motionless. These types have been thoroughly studied in Zhmur et al. (2011). In this paper, we are only interested in the periodic types, since these generate the irregular fluid advection in the surrounding flow.

Thus, we will further study passive scalar transport induced in the resulting velocity field being jointly generated by the linear shear and ellipsoidal vortex (for details see Zhmur et al., 2011),

$u=e x-\gamma y+\cos \theta \tilde{v}+\sin \theta \tilde{u}$,

$v=\gamma x-e y+\sin \theta \tilde{v}-\cos \theta \tilde{u}$,

where

$$
\begin{gathered}
\tilde{u}=-\sigma a b \tilde{c} \int_{\lambda}^{\infty} \frac{\tilde{y} \mathrm{~d} \mu}{\left(b^{2}+\mu\right) \sqrt{\xi(\mu)}}, \\
\tilde{v}=\sigma a b \tilde{c} \int_{\lambda}^{\infty} \frac{\tilde{x} \mathrm{~d} \mu}{\left(a^{2}+\mu\right) \sqrt{\xi(\mu)}},
\end{gathered}
$$

$\tilde{x}=x \cos \theta+y \sin \theta$,

$\tilde{y}=-x \sin \theta+y \cos \theta$,

and $\lambda$ determines whether a fluid particle belongs to the ellipsoidal vortex or to its surroundings. For a particle within the vortex one should take $\lambda=0$, while for a particle in the 
surrounding flow one should use the positive root, $\lambda>0$, of the equation, $\frac{\tilde{x}^{2}}{a^{2}+\lambda}+\frac{\tilde{y}^{2}}{b^{2}+\lambda}+\frac{\left(\frac{N}{f} z\right)^{2}}{\tilde{c}^{2}+\lambda}=1$.

By using Eqs. (5) and (3) one can evaluate trajectories of fluid particles within the ellipsoidal vortex and in its surroundings. Since the lengths of the vortex's semiaxes depend on time periodically (although the ellipsoid always stays ellipsoidal), the surrounding fluid undergoes a periodic perturbation inflicted by the vortex's form oscillation.

System (5) of the governing equations is a dynamical system with "one and a half degrees of freedom" (Lichtenberg and Lieberman, 1983; Zaslavsky, 1998), where an additional half degree of freedom implies a time-dependent perturbation of a stationary dynamical system. In the problem under consideration, such a time-dependent perturbation arises due to the periodic motion of the ellipsoidal vortex. So, this perturbation leads a part of trajectories of the surrounding fluid particles to exhibit chaotic behaviour (the exponential divergence of close trajectories for a finite time). Although the model allows us to study the fluid dynamics at different horizontal planes, the present study is concerned only with the dynamics of the section, $z=0$, throughout the paper. Scaling parameters of system (5) were chosen as $e=0.1, \tilde{c}=1$, and $\gamma=0$. Figure 1 shows a typical stream-line pattern of stationary system (5) with the exterior flow where the strain, $e=0.1$, is dominant over the rotation, $\gamma=0$.

System (5) is stationary when the vortex (grey zone in the figures) is motionless, and, consequently, does not change its semiaxes' lengths. The stationary system's initial values are $\frac{a(0)}{b(0)}=1.0551, \theta(0)=\frac{\pi}{4}$ (for details see Zhmur et al., 2011). When $e>\gamma$ then the exterior flow (blue lines in Fig. 1) is hyperbolic, and in the vortex vicinity, a closed recirculation zone appears (red lines in Fig. 1). The bold black curve demarcating these two motion types is a separatrix with two hyperbolic critical points. If $e<\gamma$ then the vortex rotates in the same direction as the exterior flow, hence it does not generate any specific closed recirculation zone and, therefore, no separatrix forms.

The dynamics of system (5) is regular, that is, the fluid particle trajectories coincide with stream-lines similar to those shown in Fig. 1 if the vortex is motionless, what can be achieved only by specifically choosing $a(0), b(0)$, and $\theta(0)$. However, in the general case, that is, if one chooses initial values of the parameters arbitrarily, the corresponding vortex performs periodic or aperiodic motions.

The periodic motion can be of two types, oscillation and rotation; while the aperiodic one is infinite elongation (for details see Meacham et al., 1994; Zhmur et al., 2011). In this study, only periodic motion is of interest. When oscillating or rotating, the vortex perturbs the fluid particle dynamics in its vicinity, which results in chaotic advection. Figure 2 illustrates a Poincaré section of the perturbed system (5) as $\frac{a(0)}{b(0)}=2, \theta(0)=\frac{\pi}{4}$, and the other parameters correspond to those as in Fig. 1. To construct this Poincaré section, we placed 20 markers along the line, which links the two hyper-

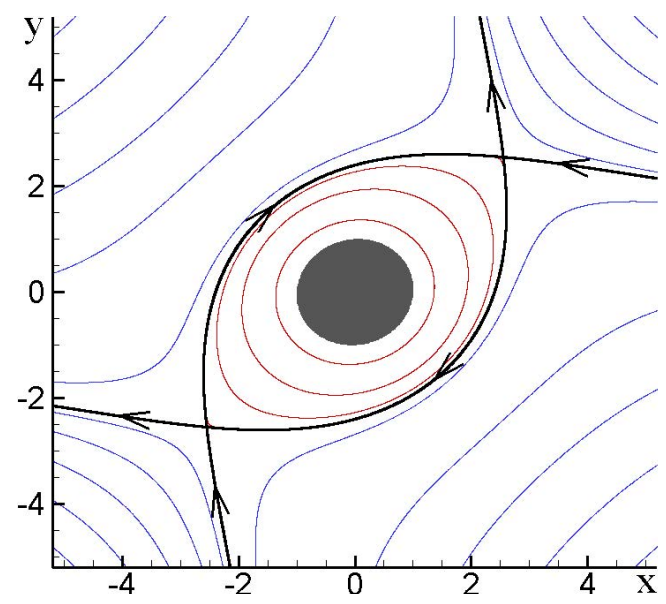

Fig. 1. A phase portrait of stationary system (5) as $e=0.1, \gamma=0$, $\frac{a(0)}{b(0)}=1.0551, \theta(0)=\frac{\pi}{4}$. The bold black curve is the separatrix dividing the closed recirculation zone (red lines) with the vortex (the grey region), and the hyperbolic exterior flow region (blue lines).

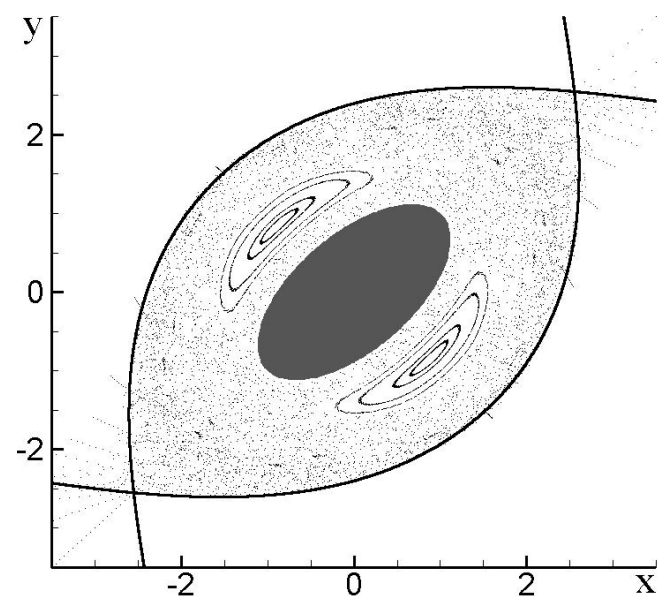

Fig. 2. A Poincaré section of system (5) as $\frac{a(0)}{b(0)}=2, \theta(0)=\frac{\pi}{4}$ with the parameters corresponding to Fig. 1 . The bold black curves are the separatrix of the associated steady state.

bolic points, and another 20 along the line, which is orthogonal to the first one in order to show the islands of regular motion, and then followed their trajectories for 30 perturbation periods. The perturbation period in this case is equal to $T=1.89166$. Further we will use this period as a characteristic timescale. We will refer to this case as the perturbed state (a detailed study of the advection generated by the perturbed system was conducted in Zhmur et al., 2011).

We should emphasize a few points that are essential to the further analysis. If the system is perturbed by the vortex motion, a large body of fluid in the vicinity of the vortex (the region filled with disordered points in Fig. 2) starts moving irregularly, that is, two close fluid particle trajectories exponentially diverge in a finite time. Also, a certain amount of the fluid (two "islands" of regular motion astride the vortex) 
is involved in regular motion (a great body of literature concerning chaotic advection in geophysical hydrodynamics can be found in Koshel and Prants, 2006; Koshel et al., 2008; Izrailsky et al., 2008).

It is worth mentioning that the trajectories of fluid particles, contained within the ellipsoidal vortex (grey region), cannot intersect the vortex's boundary. To allow such intersections to occur, we suggest implementing the diffusion process into the vortex system. Further we will show that the joint influence of the diffusion process and the irregular dynamics, namely, chaotic advection, affects the fluid particle transport differently compared to the one with diffusion and regular motion. The former we will refer to as the perturbed state case, and the latter as the steady state case.

\section{Diffusion implementation}

So, the next step is to implement a diffusivity into system (5). Therefore, consider passive scalar motion in a prescribed velocity field, $\boldsymbol{U}(\boldsymbol{r}, t)$ :

$$
\left(\frac{\partial}{\partial t}+\boldsymbol{U}(\boldsymbol{r}, t) \frac{\partial}{\partial \boldsymbol{r}}\right) q(\boldsymbol{r}, t)=\kappa \frac{\partial^{2}}{\partial \boldsymbol{r}^{2}} q(\boldsymbol{r}, t),
$$

$q(\boldsymbol{r}, 0)=q_{0}(\boldsymbol{r})$

where $q(\boldsymbol{r}, t)$ is a scalar field of a passive admixture (under certain circumstances it can be, e.g. salinity, temperature, vorticity), and $\kappa$ is the diffusivity. Expression (6) is already written in a dimensionless form.

Now, we introduce an auxiliary scalar field, $\tilde{q}(\boldsymbol{r}, t)$, being described by the following stochastic equation:

$$
\left(\frac{\partial}{\partial t}+\boldsymbol{U}(\boldsymbol{r}, t) \frac{\partial}{\partial \boldsymbol{r}}\right) \tilde{q}(\boldsymbol{r}, t)=-\boldsymbol{p}(t) \frac{\partial}{\partial \boldsymbol{r}} \tilde{q}(\boldsymbol{r}, t),
$$

$\tilde{q}(\boldsymbol{r}, 0)=q_{0}(\boldsymbol{r})$,

with $\boldsymbol{p}(t)$ being a delta-correlated vector random Gaussian process which does not depend on $\boldsymbol{U}(\boldsymbol{r}, t)$, and satisfies the conditions:

$$
\langle\boldsymbol{p}(t)\rangle=\mathbf{0},\left\langle p_{i}(t) p_{j}\left(t^{\prime}\right)\right\rangle=2 \kappa \delta_{i j} \delta\left(t-t^{\prime}\right), \quad i, j=1,2,
$$

where $\delta_{i j}$ is the Kronecker delta, $\delta(t)$ is the Dirac function, and $t, t^{\prime}$ are two consecutive instants in time.

The solution of (6) corresponds to the averaging (Klyatskin, 1994, Koshel and Alexandrova, 1999 in the solution of Eq. (7) over an ensemble of realizations of process $\boldsymbol{p}(t)$ such that

$q(\boldsymbol{r}, t)=\langle\tilde{q}(\boldsymbol{r}, t)\rangle_{p}$.

Equation (9) represents the solution of Eq. (6) in the form of a continual integral (Mesinger, 1971; Klyatskin, 1994,
2005). Now, one can solve Eq. (7) instead of Eq. (6). By exploiting the characteristics of Eq. (7), one can readily obtain

$$
\begin{aligned}
\frac{\mathrm{d}}{\mathrm{d} t} \boldsymbol{r}(t) & =\boldsymbol{U}(\boldsymbol{r}(t), t)+\boldsymbol{p}(t), \quad \boldsymbol{r}(0)=\boldsymbol{r}_{0}, \\
\frac{\mathrm{d}}{\mathrm{d} t} \tilde{q}(\boldsymbol{r}(t), t) & =0, \quad \tilde{q}(0)=q_{0}\left(\boldsymbol{r}_{0}\right) .
\end{aligned}
$$

System (10) corresponds to system (5) with $\boldsymbol{U}(\boldsymbol{r}(t), t)$ being the right-hand terms of Eq. (5), and $\boldsymbol{p}(t)$ being the diffusion term. Then, by making use of the Monte Carlo method, we take into consideration the diffusion process along the trajectory of a passive scalar (Koshel and Alexandrova, 1999).

\section{Diffusion affected transport in the steady state}

Now, we present the numerical simulation results. First, we should mention a few remarks concerning the numerical calculations. Figures 1 and 2 were initially obtained exploiting a method with an adaptive step control, namely, the BulirschStoer algorithm, that uses Richardson extrapolation with the modified middle point method. However, this method cannot be applied to calculate trajectories of a stochastic dynamical system, since the stochastic perturbation depends on the integration step. Hence, in order to correctly model a stochastic process, the integration step must be constant throughout the calculation. Therefore, to achieve an acceptable precision of our calculations, we use the Euler method with a sufficiently small integration step. Thus, first, we chose the integration step, comparing the obtained deterministic trajectories of Figs. 1 and 2 with the ones obtained by the BulirschStoer algorithm, and then, calculated the stochastic process realizations using the Euler method with the constant integration step. The step we used is $10^{-3}$, a smaller step would result in an overwhelming calculation time.

First, we have executed simulations of the diffusionaffected passive scalar transport in stationary system (10), so, the initial parameters correspond to Fig. 1. The ellipsoidal vortex in this case is motionless, so, the passive scalar advection both within the vortex and in the surroundings is regular, that is, the trajectories of passive scalars coincide with the stream lines. To study the dynamics of a scalar concentration field, we have distributed uniformly 1000 markers within the vortex region (the locus of each marker corresponds to the cell of a net covering the vortex), and then executed 1000 realizations of process $\boldsymbol{p}(t)$. Thus, the initial concentration within the vortex is 1000 markers in each cell of the uniformly distributed net covering the vortex. Then we have traced trajectories of the markers for a time interval, and memorized the positions the markers have reached.

Figure 3 shows the concentration field at the indicated time as the diffusion is equal to $\kappa=10^{-2}$, and the model's parameters correspond to Fig. 1. The bold black curve is the vortex's boundary that does not change due to stationarity. According to the figure, diffusion affects the scalar transport 


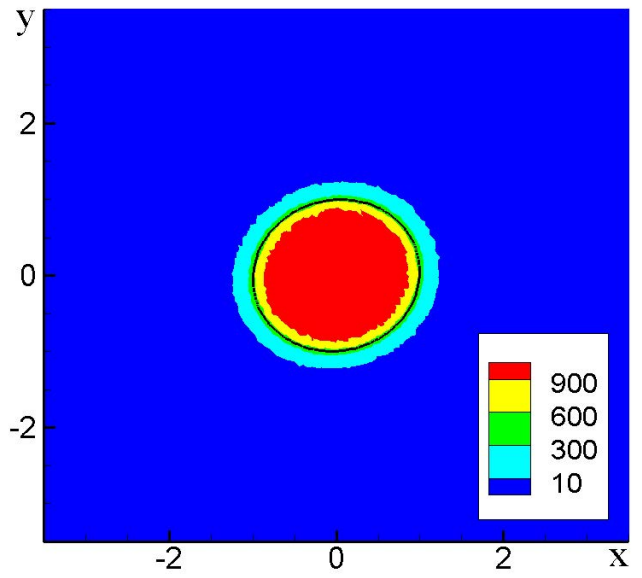

(a) $T / 4$

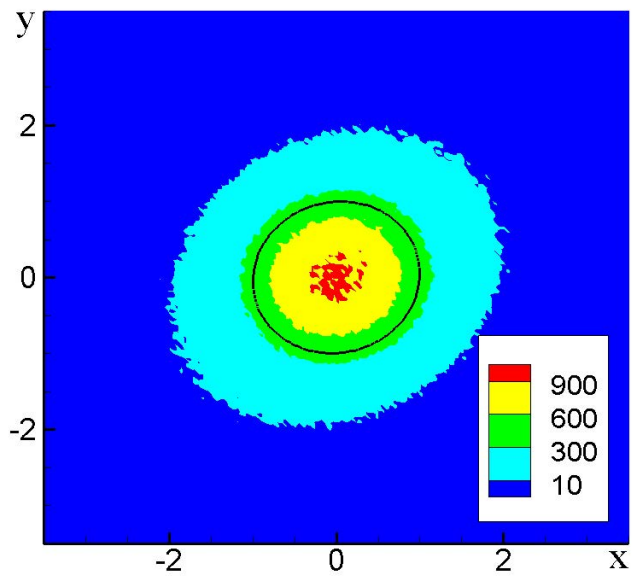

(b) $5 \cdot T$

Fig. 3. Scalar concentration field at the indicated time as $\kappa=10^{-2}$ corresponding to the parameters as in Fig. 1. The bold black curve is the vortex's boundary corresponding to the initial scalar distribution. Concentration (number of markers) is shown by colour.

weakly through slow spreading of the vortex's boundary (see Fig. 3a at time $T / 4$, where $T=1.89166$ is the characteristic period of vortex rotation). The concentration field within the vortex undergoes little changes due to diffusion, and it stays almost equal to the initial concentration filed. Since there is no source of markers, in the course of time, the concentration decreases within the vortex, and markers occupy the surrounding space (see Fig. $3 \mathrm{~b}$ at time 5.T). Hence, in the steady state, the concentration field outside the vortex spreads normally according to a Gaussian distribution.

Figure 4 illustrates the probability density of the marker distribution as a function of the values, $a$, at the $\mathrm{y}=\mathrm{x}$ line, which determine equal-area elliptic rings. In other words, Fig. 4 depicts how many markers are located within elliptic rings of same area $\Delta S$ at a given instant. All the rings belong to one elliptic family with ellipticity $\frac{a(0)}{b(0)}=1.0551$. The number of markers is normalized by the total number of markers, $\sim 10^{6}$, and by the ring area, $\Delta S=0.15$. The curves show that the marker spreading indeed progresses as a Gaussian process both within and outside the vortex. It is also of essence whether the stochastic process is significantly affected by the numerical error. To confirm that our results are stable, we calculated the discrepancy between the step we used and the step of $10^{-4}$ for the probability density function curves shown in Fig. 4. The corresponding relative error is 0.00488 . Thus, we conclude that the calculation error does not affect our results significantly.

The next part of the study is concerned with the perturbed state, when the vortex's boundary changes in time, which leads to the irregular dynamics outside the vortex.

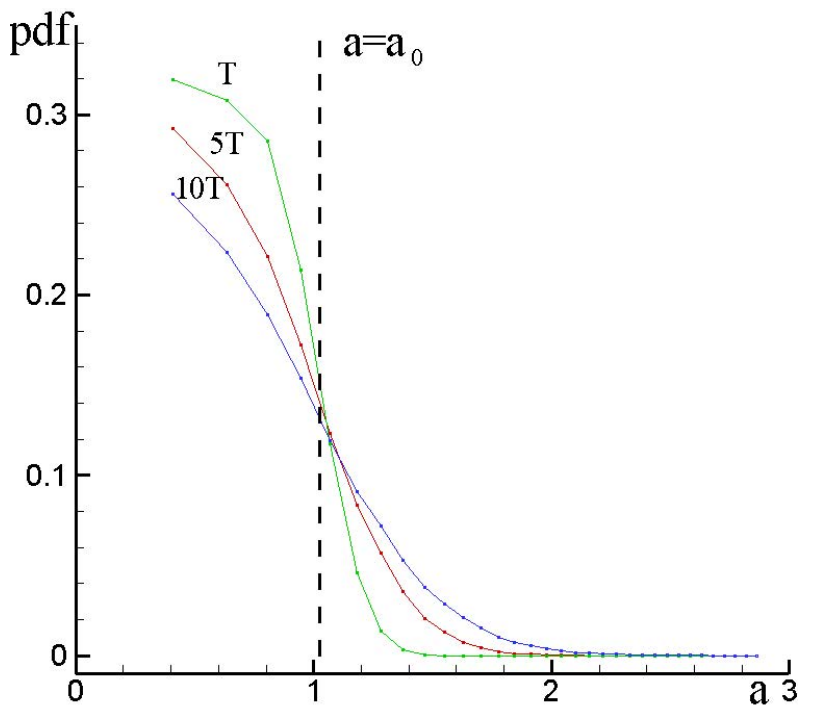

Fig. 4. Probability density as a function of the elliptic rings' major semiaxis $a$ in the steady state case. The curves depict the probability density at the instants corresponding to different number of the vortex rotation periods. Vertical dashed line marks the major semiaxis of the vortex.

\section{Diffusion-affected transport in the perturbed state}

In this part, we will analyse the diffusion-affected transport in the perturbed case, that is, when the vortex changes its semiaxes' lengths periodically. As the initial conditions we chose $\frac{\mathrm{a}(0)}{\mathrm{b}(0)}=2$, and $\theta(0)=\frac{\pi}{4}$ (see Fig. 2). With these initial conditions, the vortex rotates slightly changing its semiaxes' lengths. As opposed to the steady state, the passive scalar advection outside the vortex is now irregular, however, within the vortex, the advection is always regular. 


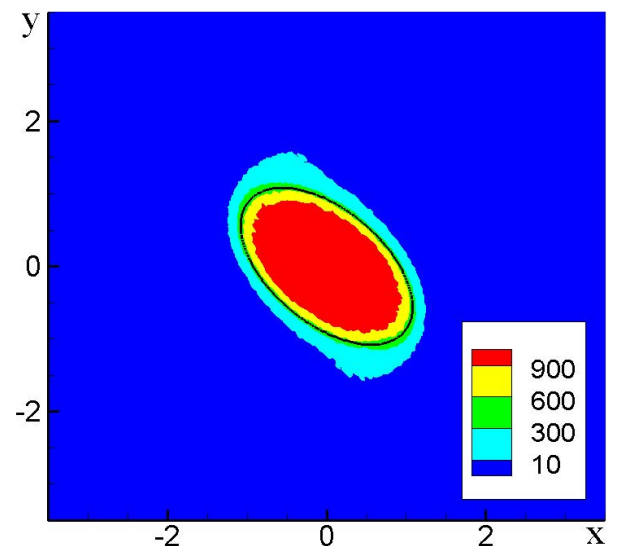

(a) $T / 4$

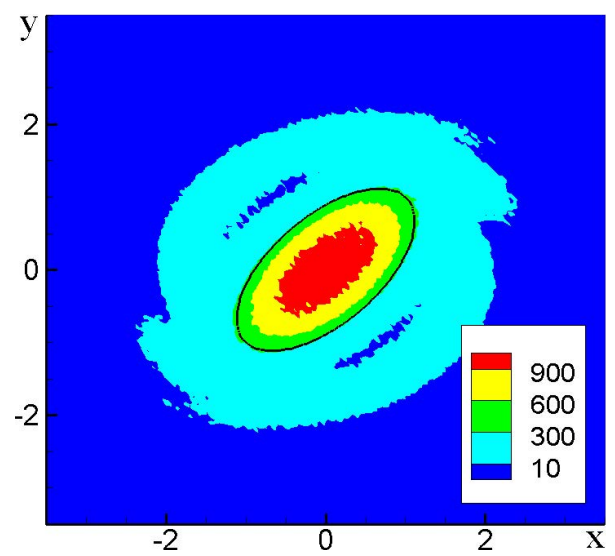

(c) $2 \cdot T$

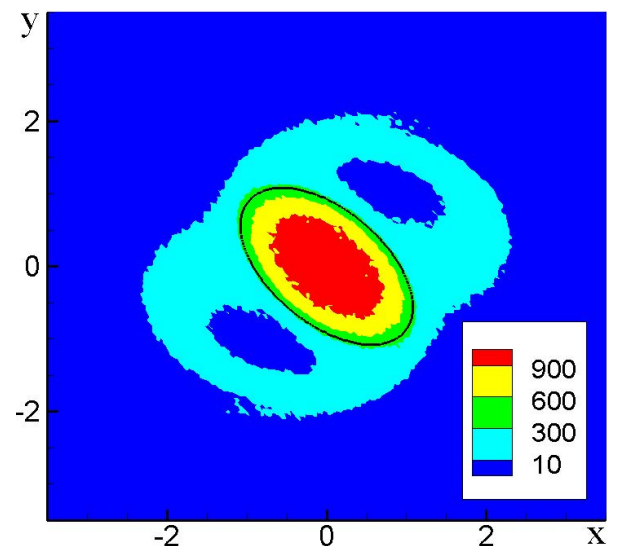

(b) $5 / 4 \cdot T$

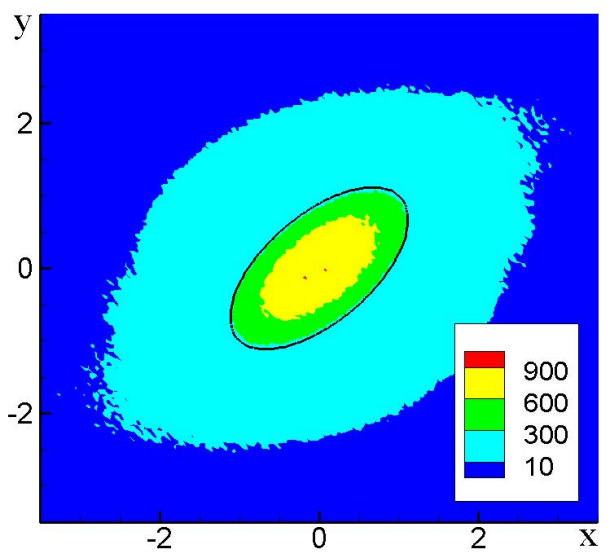

(d) $5 \cdot T$

Fig. 5. Scalar concentration field at the indicated time as $\kappa=10^{-2}$ corresponding to the parameters as in Fig. $2 \mathrm{a}$. The bold black curve is the vortex's boundary corresponding to the initial scalar distribution. Concentration (number of markers) is shown by colour.

Figure 5a, b, c, and depict the concentration field at the time corresponding to a quarter of the vortex's rotation period ( $T / 4)$, to $5 / 4 \cdot T, 2 \cdot T$, and $5 \cdot T$ rotational periods, respectively.

Now we underline a few peculiarities which can be seen in Fig. 5. First, since within the vortex the dynamics is always regular, the markers spread according to a Gaussian distribution, same as in the steady state, however, outside the vortex the markers spread otherwise due to chaotic advection. To demonstrate this explicitly we plotted Fig. 6, which shows the probability density as the corresponding rings' ellipticity is equal to $\frac{a(0)}{b(0)}=2$. In accordance with Fig. 6, within the vortex (on the left of the dashed vertical line), the marker spreading progresses the same way as in the steady state case, but outside the vortex the spreading obeys another law.

It seems that this law should be a power law, what is in agreement with the works of Jones, 1994; Rom-Kedar and Poje, 1999. This difference between the steady and perturbed states in the distribution laws outside the vortex, roughly speaking, is because, in the steady state, the probability of a marker to jump from one deterministic trajectory to another equals the probability of it to perform a reverse jump to the initial trajectory, but, in the perturbed state, these chances are not equal. In this case, if a marker jumps to another deterministic trajectory due to diffusion, this trajectory exponentially diverges from the marker's initial one, and the probability of a reverse jump to the initial trajectory decreases compared to the initial jump probability.

Second, the regions ("islands") of regular motion astride the vortex corresponding to nonlinear resonances rotate together with the vortex, and the diffused markers get into these regions much later than into the every point of the stochastic sea (see Fig. 5b, c). Within these islands, the marker spreading progresses the same way as within the vortex, namely, according to the Gaussian distribution, since no exponential divergence of close trajectories occurs.

Comparing Figs. $3 \mathrm{~b}$ and 5d, which correspond to the same instant in time, one can see that the marker spreading progresses much faster in the perturbed state. In the perturbed state the scalar concentration within the vortex is not equal 


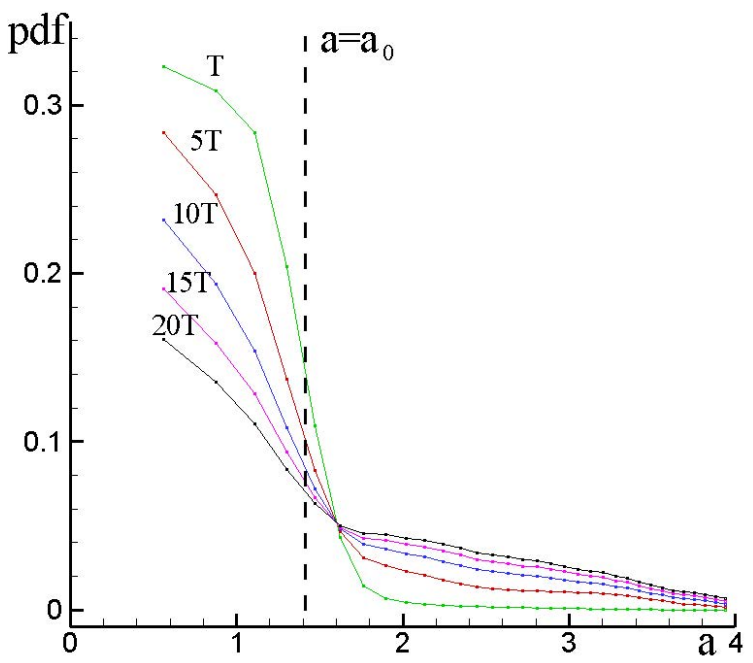

Fig. 6. Probability density as a function of the elliptic rings' major semiaxis $a$ in the perturbed state case. The curves depict the probability density at the instants corresponding to different number of the vortex rotation periods. Vertical dashed line marks the vortex's boundary.

to the initial concentration almost everywhere, while in the steady state the initial concentration remains almost the same in the vicinity of the vortex's centre. Given the length of the vortex's boundary is equal in both cases, and the boundary throughput is hence also equal, the different rate of the scalar spreading is because, in the steady state, once a marker left the vortex, it has the same probability to return inside the vortex, while, in the perturbed case, the chance of leaving the vortex is higher than the chance of returning inside it. This leads to the conclusion that the draining from the vortex is much effective in the perturbed state. Figure 7 depicts the part of markers which are located outside the vortex at instant $N_{T} \cdot T$. The green and red curves correspond to the perturbed and steady states, respectively. One can clearly see that the scalar detraining of the vortex progresses much faster in the perturbed case.

\section{Conclusions and discussion}

The cooperative impact of diffusion and advection on the scalar transport in the model of an ellipsoidal vortex embedded in a linear shear flow has been addressed. Two types of advection, namely, regular and irregular (chaotic) have been dealt with. The combined effect of the former and diffusion has been shown to result in a normal spreading of scalars outside the vortex, while with chaotic advection the transport outside the vortex seems to tend to a polynomial-law dependence. This is because chaotic advection results in the exponential divergence of close trajectories. Then, given diffusion is implemented into the system, the possibility of a scalar to jump on a close trajectory due to diffusion is not equal to the

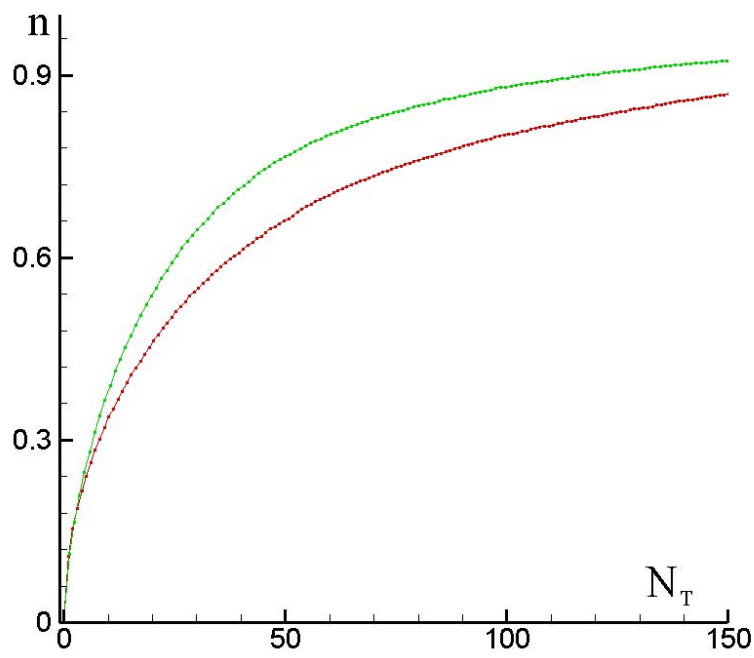

Fig. 7. The part of markers which are located outside the vortex at instant $N_{T} \cdot T$. The green and red curves correspond to the perturbed and steady states, respectively.

possibility of the scalar to jump back on the initial trajectory since the distance to this initial trajectory is $\sim e^{\lambda^{*} t^{*}}$, where $\lambda^{*}$ is the Lyapunov exponent, and $t^{*}$ is a characteristic diffusion timescale.

Comparing the steady and perturbed states which induce the regular and irregular dynamics, respectively, we have shown that the scalar transport through the vortex's boundary is more effective, i.e. the scalar emanation from the vortex happens at a higher rate, in the perturbed state. This is also caused by the exponential divergence since the probability of a scalar to return to the vortex in the perturbed state is lesser then the one in the steady state, and, hence, the vortex emanates scalars faster in the former case.

To conclude, we would like to discuss the applicability scopes of the presented study to the real ocean. In the ocean, the problem of reliable determination of the diffusivity, $K$, is quite challenging (Monin and Ozmidov, 1985; Van Dam et al., 1999; Zhurbas and Oh, 2004), however empirical estimates give an assessment, $K(l) \sim l^{4 / 3}$, where $l$ is the corresponding scale that the diffusion affects mostly. Our dimensionless diffusion parameter $\kappa$ corresponds to dimension parameter $K$ as follows: $K \sim U^{*} L^{*} \kappa$, where $U^{*}$, and $L^{*}$ are the characteristic velocity and size scales of the vortex motion. Since the governing equation of the vortex dynamics are derived by exploiting the quasi-geostrophic approximation, our characteristic parameters are to correspond to the mesoscale dynamics. Thus, by choosing the buoyancy frequency, Coriolis parameter, total depth as $N=2 \times 10^{-3} \mathrm{~s}^{-1}$, $f=10^{-4} \mathrm{~s}^{-1}, H=4 \times 10^{4} \mathrm{~m}, U^{*}=0.1 \mathrm{~m} \mathrm{~s}^{-1}, L^{*}=10^{5} \mathrm{~m}$, and $\kappa=10^{-2}$, we obtain an estimate, $K \sim 10^{2} \mathrm{~m}^{2} \mathrm{~s}^{-1}$, that according to Okubo (1971) corresponds to the apparent diffusion scale of $l \sim 10^{3} \mathrm{~m}$. 
Acknowledgements. The reported study was partially supported by RFBR, research projects: 11-05-00025-A, 12-05-31011; by The Ministry of education and science of Russia, project: 14.A18.21.0353; and by the ICMAT Severo Ochoa project SEV2011-0087.

Edited by: J. Duan

Reviewed by: three anonymous referees

\section{References}

Aref, H.: Stirring by chaotic advection, J. Fluid Mech., 143, 1-21, doi:10.1017/S0022112084001233, 1984.

Aref, H.: The development of chaotic advection, Phys. Fluids, 14, 1315-25, doi:10.1063/1.1458932, 2002.

Balasuriya, S.: An Analytical Study of General Hyperdiffusivity and Barotropic Eddies, Geo, 91, 39-62, doi:10.1080/03091920410001659290, 2004.

Balasuriya, S. and Jones, C. K. R. T.: Diffusive draining and growth of eddies, Nonlin. Processes Geophys., 8, 241-251, doi:10.5194/npg-8-241-2001, 2001.

Brown, M. G. and Samelson, R. M.: Particle motion in vorticityconserving, two-dimensional incompressible flows, Phys. Fluids, 6, 2875-2876, doi:10.1063/1.868112, 1994.

Chelton, D. B., Schlax, M. G., Samelson, R. M., and de Szoeke, R. A.: Global observations of large oceanic eddies, Geophys. Res. Lett., 34, L15606, doi:10.1029/2007GL030812, 2007.

Chelton, D. B., Schlax, M. G., and Samelson, R. M.: Global observations of nonlinear mesoscale eddies, Prog. Oceanogr., 91, 167-216, doi:10.1016/j.pocean.2011.01.002, 2011.

Dahleh, M. D.: Exterior flow of the Kida ellipse, Phys. Fluids AFluid, 4, 1979-1985, doi:10.1063/1.858366, 1992.

Izrailsky, Y. G., Koshel, K. V., and Stepanov, D. V.: Determination of the optimal excitation frequency range in background flows, CHAOS, 18, 013107, doi:10.1063/1.2835349, 2008.

Jones, S. W.: Interaction of Chaotic Advection and Diffusion, Chaos Soliton. Fract., 4, 929-940, doi:10.1016/0960-0779(94)90132-5, 1994.

Kida, S.: Motion of an elliptic vortex in a uniform shear flow, J. Phys. Soc. Jpn., 50, 3517-3520, doi:10.1143/JPSJ.50.3517, 1981.

Klyatskin, V. I.: Statistical description of the diffusion of a passive tracer in a random velocity field, Phys.-Usp.+, 37, 501-513, doi:10.1070/PU1994v037n05ABEH000110, 1994.

Klyatskin, V. I.: Stochastic Equations through the Eye of the Physicist (Basic Concepts, Exact Results, and Asymptotic Approximations), Elsevier Science, vii-viii, doi:10.1016/B978-0444517975/50000-9, 2005.

Koshel, K. V. and Alexandrova, O. V.: Some results of a numerical modeling of the diffusion of passive tracers in a random field of velocities, Izv. Atmos. Ocean. Phys., 35, 578-588, 1999.
Koshel, K. V. and Prants, S. V.: Chaotic advection in the ocean, Phys.-Usp.+, 176, 1177-1206, doi:10.1070/PU2006v049n11ABEH006066, 2006.

Koshel, K. V., Sokolovskiy, M. A., and Davies, P. A.: Chaotic advection and nonlinear resonances in an oceanic flow above submerged obstacle, Fluid Dyn. Res., 40, 695-736, doi:10.1016/j.fluiddyn.2008.03.001, 2008.

Lichtenberg, A. and Lieberman, M.: Regular and Stochastic Motion, Springer-Verlag, New York, 1983.

Meacham, S., Pankratov, K. K., Shchepetkin, A. F., and Zhmur, V. V.: The interaction of ellipsoidal vortices with background shear flows in a stratified fluid, Dynam. Atmos. Oceans, 21, 167212, doi:10.1016/0377-0265(94)90008-6, 1994.

Mesinger, F.: Numerical integration of the primitive equations with a floating set of computation points: experiments with a barotropic global model, Mon. Weather Rev., 99, 15-29, doi:10.1175/1520-0493(1971)099<0015:NIOTPE > 2.3.CO;2, 1971.

Monin, A. and Ozmidov, R.: Turbulence in the Ocean, Reidel, Dordrecht, 1985.

Okubo, A.: Oceanic diffusion diagrams, Deep-Sea Res., 18, 789_ 802, 1971.

Pedlosky, J.: Geophysical Fluid Dynamics, 2nd Edn., Springer, New York, 1987.

Polvani, L. and Wisdom, J.: Chaotic Lagrangian trajectories around an elliptical vortex parch embedded in a constant and uniform background shear flow, Phys. Fluids A-Fluid, 2, 123-126, doi:10.1063/1.857814, 1990.

Rom-Kedar, V. and Poje, A. C.: Universal properties of chaotic transport in the presence of diffusion, Phys. Fluids, 11, 2044 2057, doi:10.1063/1.870067, 1999.

Van Dam, G. C., Ozmidov, R. V., Korotenko, K. A., and Suijlen, J. M.: Spectral structure of horizontal water movement in shallow seas with special reference to the North Sea, as related to the dispersion of dissolved matter, J. Marine Syst., 21, 207-228, doi:10.1016/S0924-7963(99)00015-9, 1999.

Zaslavsky, G.: Physics of Chaos in Hamiltonian Dynamics, Imperial College Press, London, 1998.

Zhmur, V. V.: Localized eddy formation in a shear-flow, Oceanology, 28, 536-538, 1988.

Zhmur, V. V.: Subsurface mesoscale eddy structures in a stratificated ocean, Oceanology, 29, 28-32, 1989.

Zhmur, V. V. and Pankratov, K. K.: The dynamics of the semi-ellipsoid subsurface vortex in the non-uniform flow, Okeanologiya, 29, 205-211, 1989.

Zhmur, V. V., Ryzhov, E. A., and Koshel, K. V.: Ellipsoidal vortex in a nonuniform flow: Dynamics and chaotic advections, J. Mar. Res., 69, 435-461, doi:10.1357/002224011798765204, 2011.

Zhurbas, V. and Oh, I. S.: Drifter-derived maps of lateral diffusivity in the Pacific and Atlantic Oceans in relation to surface circulation patterns, J. Geophys. Res., 109, C05015, doi:10.1029/2003JC002241, 2004. 